\title{
Relire la pensée de Frei Otto sur l'espace et les formes à la lumière du numérique (et réciproquement)
}

\section{Revisiting Frei Otto's thoughts on space and forms in the light of digital experiments (and vice versa)}

\author{
Marie-Pascale Corcuff ${ }^{*}$ \\ GRIEF, ENSAB, 35000 Rennes, France
}

\begin{abstract}
Résumé. Frei Otto a toute sa vie expérimenté sur l'espace et les formes, à travers des expériences de pensée ou des dispositifs matériels. Son attitude rejoint celle qui préside à l'écriture d'algorithmes génératifs et la confrontation des deux démarches s'avère stimulante pour l'imagination créatrice. Cet aller-retour est illustré à travers trois cas d'étude qui concernent la délimitation de territoires, les forces de distanciation/attraction, et enfin les chemins optimisés.
\end{abstract}

Mots-clés. Expérimentation, Espace/Forme, Frei Otto, Processus génératifs

\begin{abstract}
Frei Otto has experimented on space and forms all along his life, through thought experiments and physical devices. His way of thinking may be linked to what is at the core of writing generative algorithms, and confronting both approaches is a stimulant for creative imagination. This two-way look is illustrated by three study cases, concerning territory delimitation, distancing/attracting forces, and optimized paths.
\end{abstract}

Keywords. Experimentation, Space/Form, Frei Otto, Generative Processes

\section{Introduction}

Frei Otto est renommé comme architecte expérimentateur et sa recherche sur les formes optimales, à partir de modèles en films de savon par exemple, est la facette la plus connue de son activité exploratoire. Mais en 2009 est paru un ouvrage (Otto 2009) rendant compte d'une pensée sur l'espace plus fondamentale, et relatant des expériences, expériences de

* marie-pascale.corcuff@rennes.archi.fr 
pensée et expériences matérielles, menées par Frei Otto bien avant cette date de parution. Ce livre modeste (112 pages), illustré de croquis rapides et de photos en noir et blanc montrant des dispositifs expérimentaux peu sophistiqués, et ne mentionnant pas une seule fois l'existence de l'informatique, s'est révélé rejoindre bien des réflexions liées à l'élaboration de processus algorithmiques génératifs. Nous envisageons le numérique ici, non comme un outil de représentation des formes architecturales, mais comme un outil d'exploration et de simulation de phénomènes engendrés par un système de règles, en particulier les formes, les configurations, rencontrées dans la nature. Le dialogue s'instaure dans les deux sens: les expériences de Frei Otto peuvent donner des idées pour des expériences numériques, mais, inversement, ses expériences peuvent être éclairées par nos explorations algorithmiques. Cette confrontation mène à des réflexions fondamentales sur l'espace et les formes. L'objectif de Frei Otto explicité par le sous-titre de l'ouvrage concerne le domaine que l'on nomme généralement urbanisme, et la plupart des propositions d'application qu'on y trouve relèvent effectivement de cette échelle de conception, jusqu'à un paragraphe intitulé "Thoughts on the ideal city ». Au-delà de cela, il nous semble que les réflexions menées par Otto sont bien plus générales et fondamentales, et qu'en définitive les applications ne sont pas ce qu'il y a de plus important, ou plutôt que l'on ne peut prédire quelles applications pourront en être déduites, à quelque échelle que ce soit. L'ouvrage de Frei Otto comporte deux parties : A. Processes of Occupation et $B$. Processes of Connection. La première partie décrit la constitution de territoires, la seconde celle de chemins, le mot le plus important dans les deux cas étant celui de processus. Nous avons extrait de l'ouvrage trois cas d'étude pour lesquels nous confrontons la démarche d'Otto à notre propre démarche de recherche d'algorithmes génératifs.

\section{Trois cas d'étude}

\subsection{Délimitation de territoires}

Au tout début de son exploration des processus d'occupation, Frei Otto expose la notion de cellule d'occupation, et décrit la démarcation de territoires adjacents (dont les centres sont distribués «aléatoirement», nous reviendrons sur la notion de distribution dans le point suivant) ainsi : «One demarcates the territory of an object by the perpendicular bisectors of the nearest points », et dessine un croquis (fig. 1).

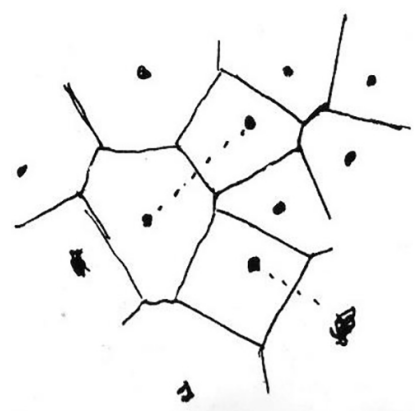

Figure 1. Délimitation de territoires (Otto 2009, p. 10)

Il est frappant de constater qu'Otto semble découvrir naïvement un concept pourtant bien connu et ancien : il s'agit d'un diagramme de Voronoï, ou encore d'un pavage de Dirichlet, ou, si l'on est plutôt géographe que mathématicien, de polygones de Thiessen. Il 
est vrai qu'avec d'aussi nombreux pères putatifs, cette construction si simple et naturelle peut tout aussi bien être revendiquée par Frei Otto.

Quoi qu'il en soit, Otto revient sur cette formation de territoires adjacents un plus loin d'une manière plus intéressante, en termes de processus. Sa première image est celle de graines qui tombent en certains points (dans toute cette partie Otto envisage une distribution aléatoire des "germes»), les plantes se développant à partir de ces germes en cercles concentriques à vitesse égale, sans que les territoires propres à chaque germe se chevauchent (fig. 2).
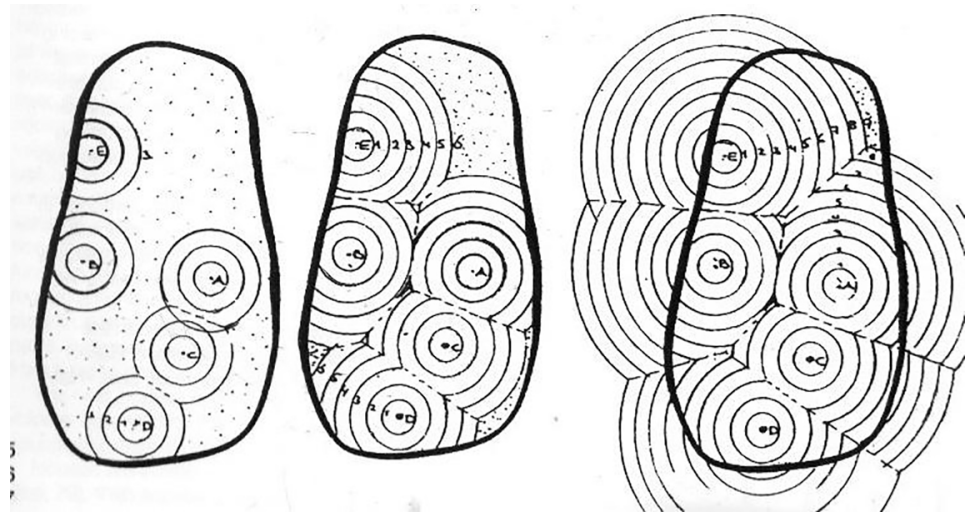

Figure 2. Croissance de territoires (Otto 2009 p. 32)

Il s'agit clairement d'une expérience de pensée, peu de plantes se développant de cette manière en réalité. Otto passe ensuite à une expérience plus concrète, qu'il dit avoir menée

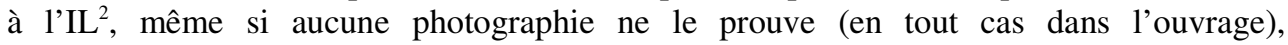
l'expérience n'étant illustrée que par quelques croquis. Il s'agit du « sand flow apparatus », un «bac à sable» dont le fond comporte des trous à travers lesquels le sable s'écoule (fig. 3 ). Cela produit un relief, constitué de cratères coniques qui s'intersectent pour former des lignes de crêtes, qui, vues de dessus, reproduisent effectivement les cellules de Voronoï.

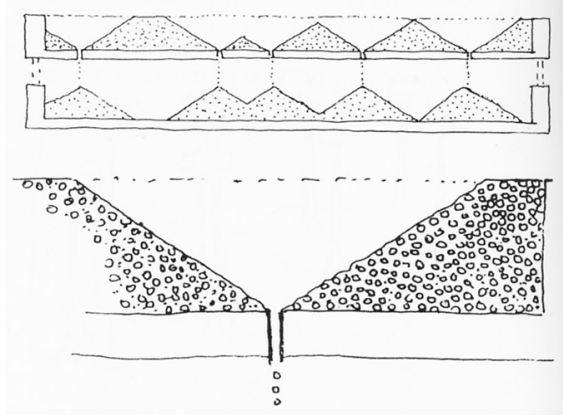

Figure 3. Bac à écoulement de sable (Otto 2009 p. 32)

Si l'on ne trouve pas de photographies du dispositif et des reliefs de sable produits par Frei Otto, il est possible par contre de voir sa mise en œuvre réalisée récemment par d'autres chercheurs et étudiants (http://drl.aaschool.ac.uk/portfolio/35-degree/). Ces expérimentateurs vont jusqu'à solidifier le fragile relief de sable pour en faire une maquette architecturale. On peut aussi mentionner l'interprétation à l'échelle architecturale de Victor

${ }^{2}$ Institut für Leichtbau Entwerfen und Konstruieren, Universität Stutgart 
Jorgensen (https://archive.dpa-etsam.com/projects/otto-drops), et les œuvres de JeanBernard Métais (en particulier « Temps imparti », 1999-2002 :

http://www.jbmetais.com/index.php?act=article\&id=22).

Nous avions suivi une cheminement de pensée assez différent, quelques années avant la parution du livre, en imaginant de donner aux pixels d'un bitmap un niveau de gris correspondant à la distance de chaque pixel au plus proche d'un ensemble de «sites » donnés. Nous avions appelé cette représentation « distance map », ou « carte de distance » (Corcuff 2005) et exploré des variantes de représentations (fig. 4), comme d'assigner au pixel une couleur correspondant au site le plus proche, ce qui produit de véritables diagrammes de Voronoï. Une autre variante a consisté à attribuer au pixel un niveau de gris proportionnel au sinus de la distance, ce qui fait ressembler l'image aux cercles concentriques d'Otto. Une dernière représentation, «naturelle» puisqu'il s'agit d'une « carte», consiste à traduire le niveau de gris en altitude, ce qui conduit à des reliefs identiques à ceux obtenus par l'écoulement du sable dans le dispositif de Frei Otto. Ceci montre que, contrairement à ce que laisse penser l'expérience, le modèle d'écoulement du sable n'est pas un processus. C'est bien une carte, tout est donné à l'avance, l'altitude $\mathrm{z}$ en chaque point du relief est donnée par sa position en $(\mathrm{x}, \mathrm{y})$ et la pente déterminée par le type de grains.

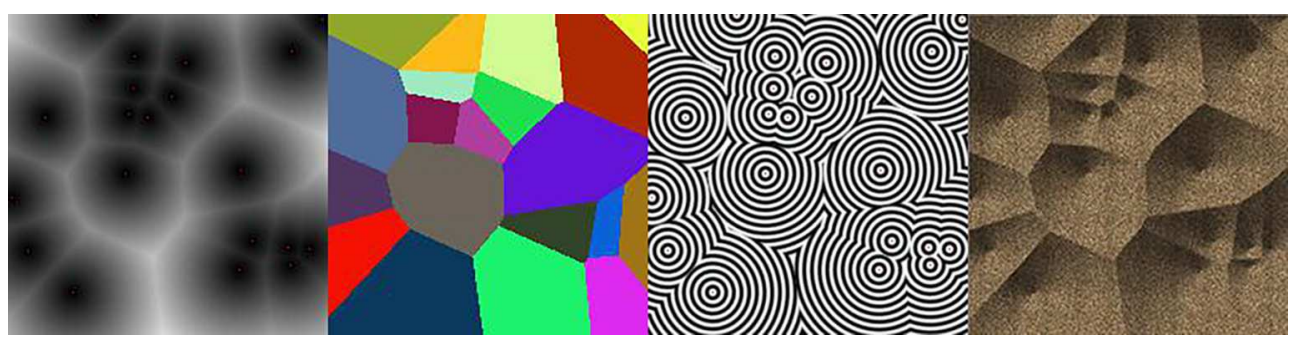

Figure 4. Cartes de distance (Corcuff 2005)

Frei Otto nous a amené à introduire deux variantes auxquelles nous n'avions pas pensé. Il propose d'abord d'échelonner dans le temps la semence des germes (dans son modèle de croissance végétale), ou l'écoulement du sable (en remontant l'ouverture du fond du bac), Les territoires ne sont alors plus des polygones, les frontières s'incurvent. L'autre variante consiste à considérer un taux de croissance différent pour chaque germe. Dans ce cas il n'y a pas d'équivalent pour le sable, qui s'écoule inexorablement de la même façon partout (fig. $5) \ldots$
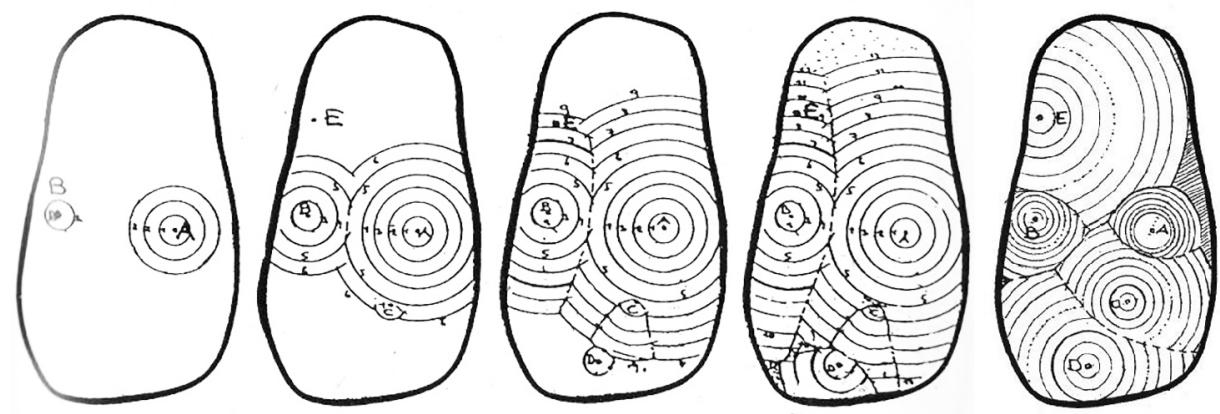

Figure 5. Croissances à départ différé / à vitesses différentes (Otto 2009 p. 33, 34) 
Ceci se traduit dans le modèle numérique par l'ajout à la distance d'une constante (propre à chaque site) pour le premier cas, et par la multiplication de la distance par un facteur (propre à chaque site) dans le second. Les résultats de la mise en œuvre de ces algorithmes sont très analogues à ceux qu'Otto a esquissés (fig. 6).

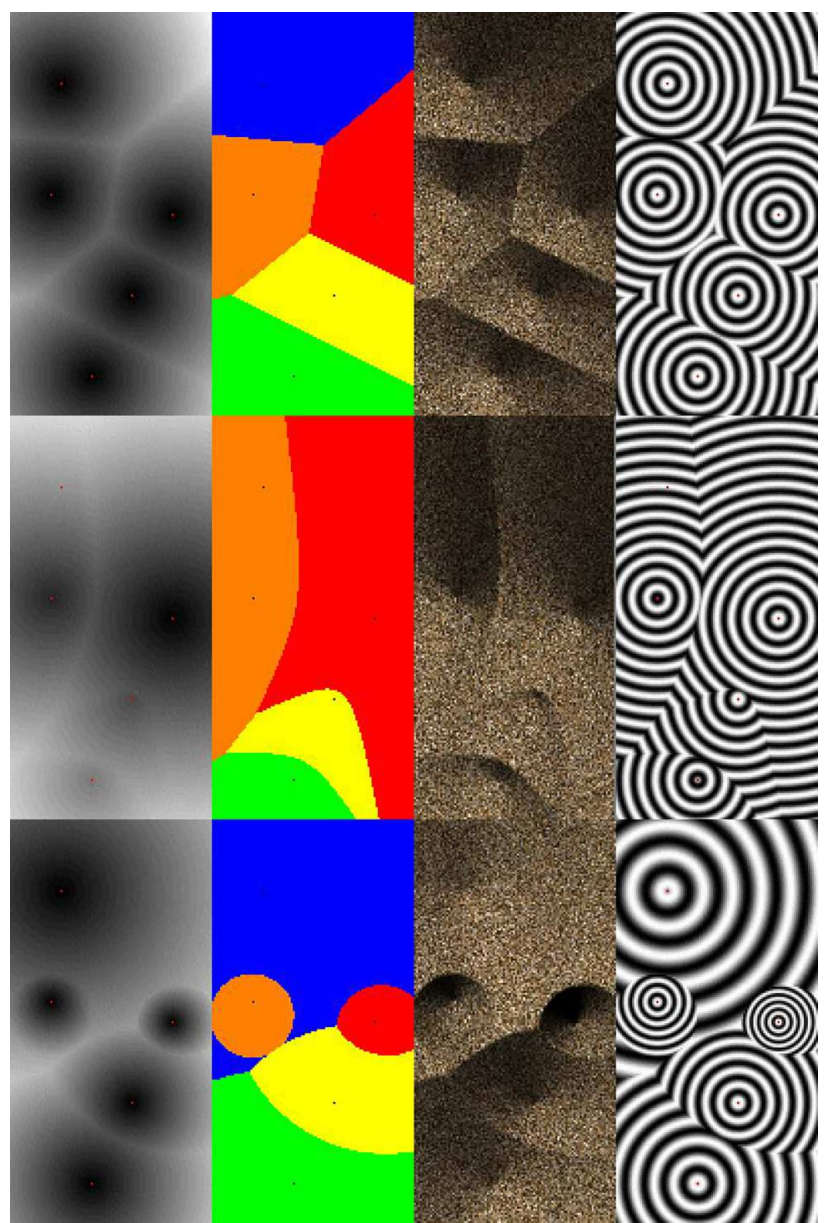

Figure 6. Cartes de distance : normales, avec ajout de constantes, avec multiplication par facteurs (Corcuff 2011)

Nous avons insisté sur le fait que ce modèle n'est pas un processus an sens propre, cependant, ce modèle de constitution de territoire peut être envisagé comme un processus. C'est un peu ce que tente Otto avec sa métaphore de croissance de plantes. Mais l'idée est plus parlante si l'on évoque la propagation de feux, par exemple, à partir d'un certain nombre de points de départ. Nous avons donc étudié la question à l'aide de modèles qui sont effectivement des processus: des automates cellulaires dont certaines variantes peuvent simuler une croissance concentrique, un modèle d'agrégation limitée par la diffusion (DLA), et enfin un modèle inverse de ce dernier qui consiste à faire partir des particules d'un centre et de les agréger dès qu'elles rencontrent l'extérieur de l'amas en formation, et que nous appelons «prolifération». Si l'on applique ces modèles à une configuration de sites similaire à celle employée pour les cartes de distance, et sans changer les règles, le résultat est analogue. Mais la force des modèles numériques, c'est que l'on 
peut plus facilement jouer avec les règles... À partir du modèle de prolifération, le changement de règle consistant à partir, non plus des centres donnés, mais de n'importe quel point des amas en formation, change tout: les territoires ne sont plus traités démocratiquement, et ceux qui, par hasard, croissent les premiers ont de plus en plus de chance de croître et, au bout d'un certain temps, dominent les autres (Corcuff 2011).

\subsection{Distanciation et attraction}

Avant d'aborder la question traitée ci-dessus, Frei Otto s'interroge sur les « forces » en présence dans l'occupation de l'espace par des éléments considérés comme ponctuels. Elles sont de deux types : la distanciation (ou répulsion) et l'attraction.

Il examine d'abord la distanciation, qui intervient lorsque les éléments cherchent à être le plus loin possible les uns des autres, et aboutit à une optimisation de l'occupation de l'espace en termes de minimisation de la promiscuité. Otto l'observe dans la façon dont des prédateurs marquent leur territoire, aussi bien que dans les territoires formés par les chasseurs, les trappeurs, ou les exploitants de forêts ou autres. Une première version du phénomène ne permet pas aux éléments déjà installés de bouger, une seconde consiste à permettre aux occupants de se réarranger dès qu'un nouveau arrive. C'est cette variante qu'Otto met en œuvre avec le dispositif expérimental suivant: des épingles aimantées flottent (grâce à un petit morceau de polystyrène) à la surface d'une cuvette d'eau, toutes orientées dans le même sens (fig. 7). Elles se repoussent donc les unes les autres. Elles sont arrêtées par le bord d'une plaque découpée en divers types de formes : patatoïde, triangle, rectangle, cercle, etc. Les épingles sont ajoutées une à une, et tendent (plus ou moins) à se disposer suivant une grille régulière triangulaire, les territoires constituant pour leur part le pavage dual, c'est-à-dire un pavage hexagonal (fig. 8). Ceci n'a rien d'étonnant, c'est effectivement le pavage optimal, et il se rencontre dans maintes configurations naturelles.
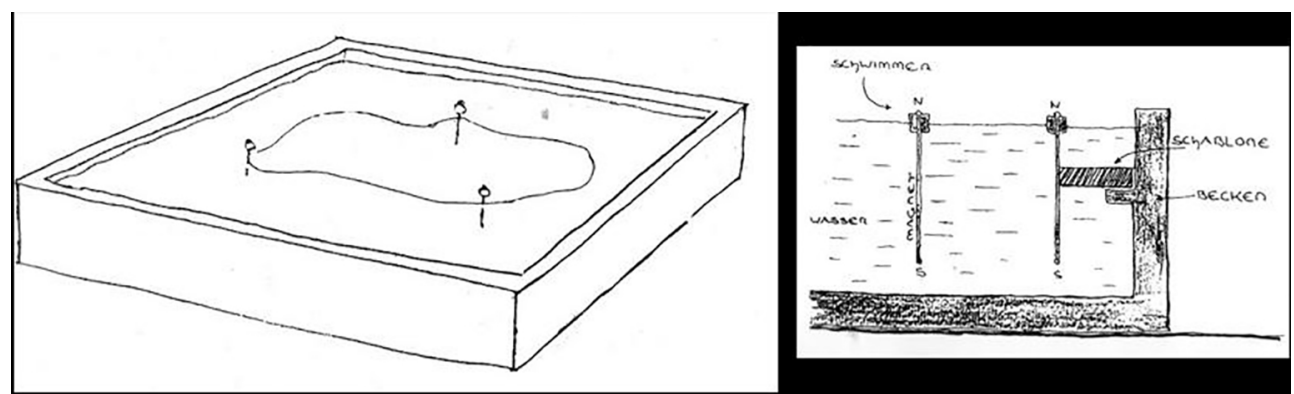

Figure 7. Dispositif pour la distanciation (Otto 2009 p. 16)
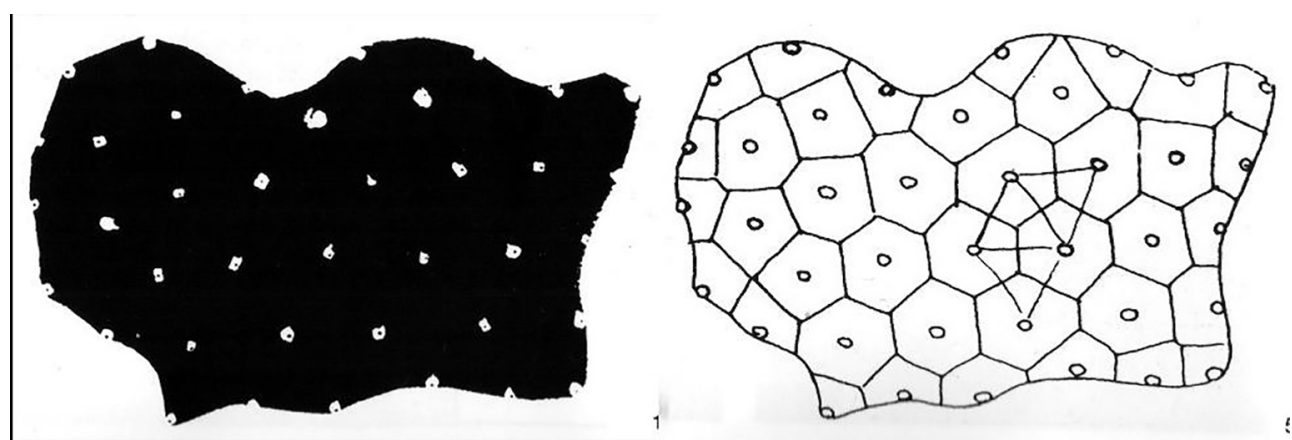

Figure 8. Résultat de la distanciation (Otto 2009 p. 24) 
Ce problème est aussi l'un des premiers que l'on rencontre dans une approche algorithmique. C'est le premier exemple du livre de Paul Coates (Coates 2010), comme témoin d'une approche «bottom-up » versus «top-down». On peut tout à fait calculer les coordonnées d'une grille triangulaire et y placer les éléments (approche top-down). Mais une approche plus intéressante est de placer les éléments n'importe où et de les laisser se réarranger eux-mêmes par une simple répulsion (ou distanciation). On peut calculer la force de répulsion exercée sur un élément par tous les autres, ou bien on peut faire encore plus simple, en faisant s'écarter d'un pas chaque élément de son plus proche voisin ( $\min / \mathrm{max}$ ). Quelle que soit la méthode, on aboutit à des configurations similaires à celles obtenues par Otto, mises en lumière par le calcul d'une carte de distance (fig. 9).

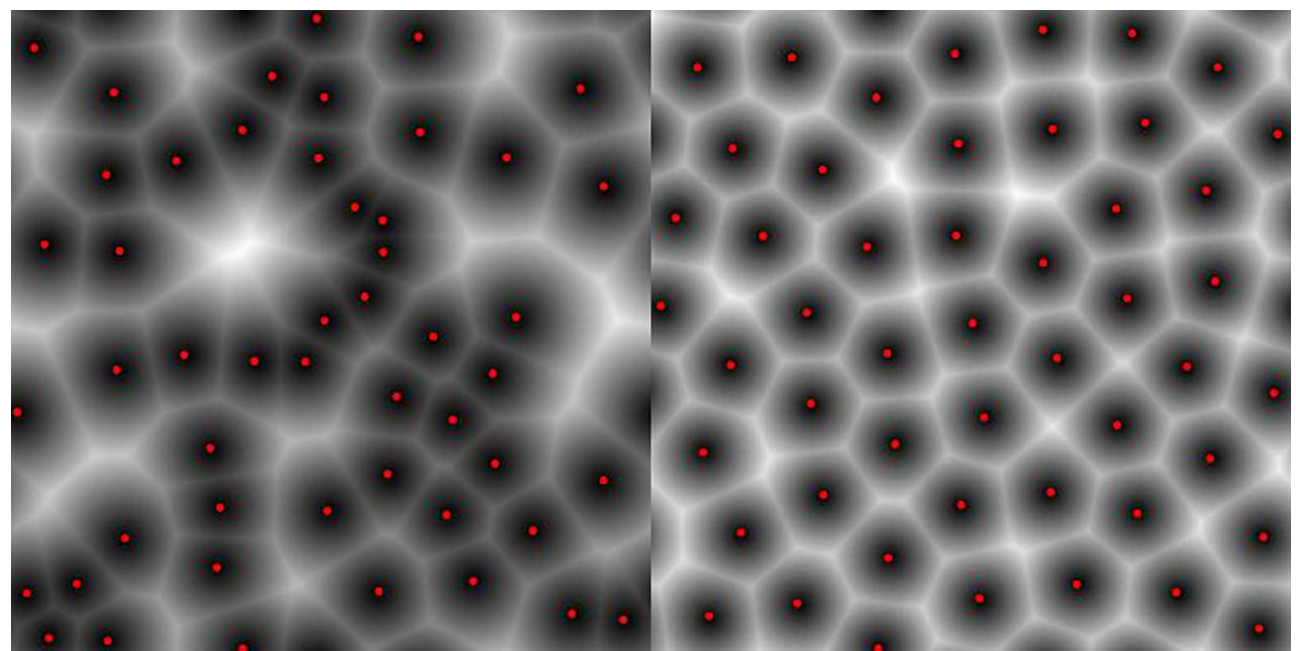

Figure 9. Algorithme de distance $\min / \max$ (Corcuff 2011)

Cet exercice permet d'expliciter une nouvelle loi de l'espace : certes la configuration optimale est une grille triangulaire duale d'un pavage hexagonal régulier, mais si l'espace (en l'occurrence le plan) n'est pas infini, il faut savoir que l'on n'obtiendra jamais cette configuration (Corcuff et alii 2014).

S'il y a des phénomènes de distanciation, il y a aussi des phénomènes d'attraction, dans la nature ou dans les installations humaines. Otto observe ce phénomène d'abord avec des bulles de savon à la surface d'une cuvette d'eau, puis tente de combiner ce rapprochement des bulles avec l'écartement des épingles aimantées. Malheureusement la force exercée par les bulles est trop forte et contrecarre l'effet magnétique. Otto utilise alors des miettes de polystyrène qui sont attirées par les flotteurs (eux-mêmes en polystyrène) par électricité statique. Petit à petit les miettes s'agglutinent autour des épingles en formant des « colonies se développant organiquement » selon Otto (fig. 10).
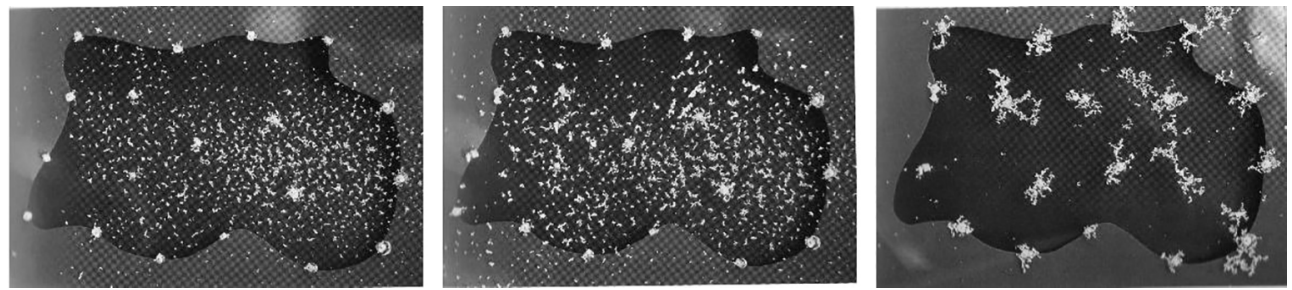

Figure 10. Dispositif pour l'attraction (Otto 2009 p. 45) 
De nouveau il est amusant de voir Otto redécouvrir «naïvement» un processus très général, qu'en informatique on nomme «DLA » (agrégation limitée par la diffusion). Il s'agit d'un véritable processus, qui ne peut s'obtenir que pas à pas. La situation de départ consiste en une cellule unique, puis des cellules se déplacent aléatoirement, jusqu'à rencontrer une cellule de l'amas en formation. En principe, il existe des règles concernant la distance à laquelle faire partir les cellules, mais en fait la configuration n'est pas essentiellement différente si on fait partir les cellules d'un point quelconque comme l'imagine Otto (fig. 11).
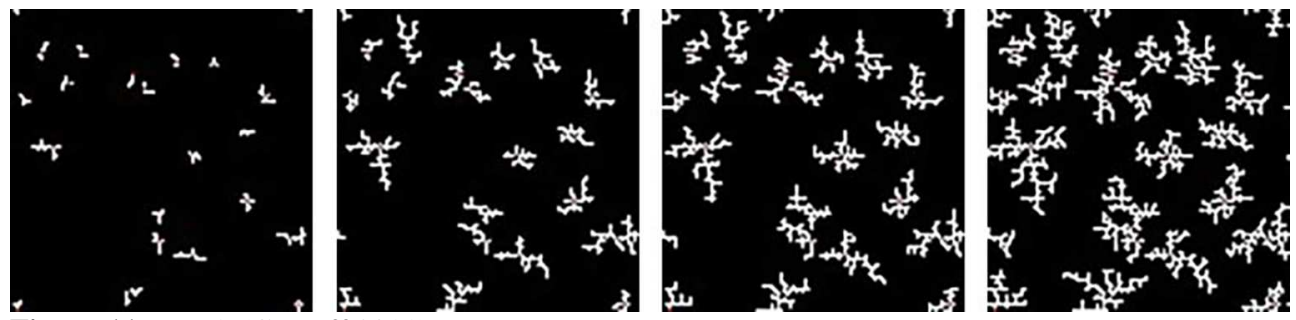

Figure 11. DLA (Corcuff 2011)

Notons que ces modèles de type DLA ont été effectivement très largement développés en urbanisme, en particulier par Michael Batty et Paul Longley (Batty \& Longley 1994). Mais, là encore, Otto ne fait pas allusion à ces recherches dans son ouvrage.

\subsection{Chemins optimisés}

Otto consacre une bonne partie de la seconde section de son livre à la question des chemins optimisés. Entre un certain nombre de points, on peut considérer les réseaux de chemins directs, qui relient tous les points deux à deux, et les réseaux de chemins minimaux, qui optimisent la longueur globale de parcours. Dans ce deuxième cas, le temps de trajet de chaque liaison de point à point est augmentée, mais la quantité d'asphalte qui serait nécessaire pour l'ensemble des chemins est minimale, ainsi d'ailleurs que le carburant requis pour un itinéraire qui relierait tous les points du système. Le résultat est un graphe qui ne comporte pas de boucle, c'est donc un arbre, et tous les embranchements (pattes d'oie) se font suivant un angle de $120^{\circ}$. Dans certains cas, cet arbre peut être unicursal (sans branches). Enfin, à partir de quatre points, le système minimal n'est pas forcément unique : par exemple, pour les sommets d'un carré, il y a deux configurations alternatives. Notons encore à partir de cet exemple que le système minimal rompt la symétrie initiale de la configuration.

Cette considération d'économie globale est matérialisée physiquement par des films de savon reliant des épingles fixées sur une plaque de verre ou de plexiglas : il s'agit là d'une minimisation de l'énergie. Otto a réalisé de telles expériences, et les photographies en sont bien connues. Mais cette résolution idéale, pour élégante qu'elle soit, ne le satisfait pas totalement, car elle impose des détours parfois importants entre des points pourtant proches. Il oppose cette optimisation drastique à une optimisation qui permet les boucles, et surtout minimise les détours, tout en essayant de diminuer la longueur totale. Cette approche ne peut qu'être heuristique : contrairement aux systèmes de chemins minimaux, qui ont une résolution assurée (même si multiple), il n'y a pas d'équation, ou même d'algorithme, menant à une solution unique.

Pour explorer cette question, Frei Otto et Marek Kolodziejczyk, à l'IL, ont introduit un autre modèle très connu, celui de points reliés par des brins de laine auxquels on donne une certaine laxité avant de les mouiller, ce qui a pour effet de les faire se rétracter et 
s'agglomérer. L'une de ces expériences dont on rencontre le plus souvent l'image concerne une configuration de 24 points sommets d'un polygone régulier (fig. 12). Ce type de configuration est intéressant, car c'est un exemple limite où le système minimal serait constitué du polygone lui-même, moins une arête, un système donc très inefficace en termes d'évitement des détours.
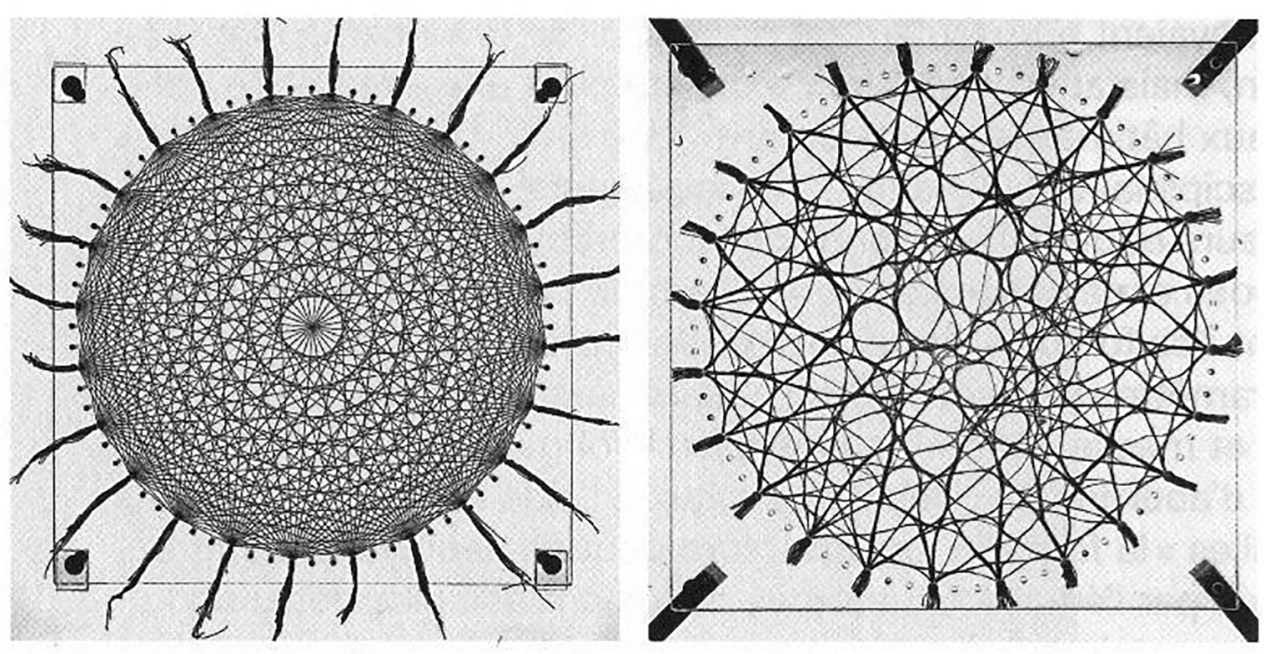

Figure 12. Dispositif pour la recherche de chemins optimisés : sans/avec laxité (IL 1991)

Nous avons atteint un résultat analogue par un moyen a priori très différent, qui se réfère au comportement des fourmis. Le modèle mis en œuvre est très simple : étant donnés un certain nombre de sites, une «fourmi » quitte l'un d'entre eux et se dirige vers un autre, au hasard ; elle laisse sur son chemin des «phéromones », i. e. les points de son parcours sont stockés dans une mémoire. Arrivée à bon port, elle repart vers une autre destination tout aussi aléatoire ; mais à présent les phéromones présentes dans son voisinage influent sur sa trajectoire. Dans cette version très peu sophistiquée, les phéromones ont toutes le même poids, mais seulement un nombre fixe d'entre elles est conservé : dès qu'une phéromone est ajoutée, la plus ancienne est supprimée. Cela permet de prendre en compte la non pérennité des phéromones. Le premier paramètre de cet algorithme est ce nombre de phéromones (pherohist), l'autre étant le voisinage dans lequel ces phéromones peuvent agir (vois). Nous avons appliqué ce modèle à des configurations diverses de points, en particulier les sommets de polygones réguliers. La figure montre le résultat de la mise en œuvre de cet algorithme pour les 24 sommets d'un polygone régulier (fig. 13). La comparaison avec les résultats de Frei Otto est frappante, d'autant que l'on peut jouer sur les paramètres (pherohist et vois) de la même façon qu'Otto jouait sur le degré de laxité. 


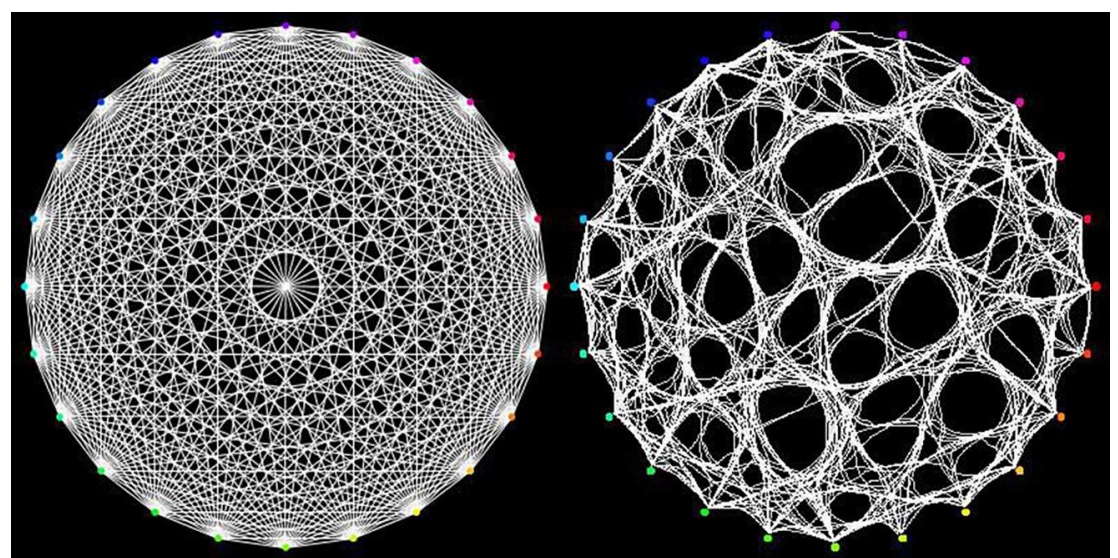

Figure 13. Algorithme fourmis/phéromones : vois $=0$, pherohist $=0$ / vois $=15$, pherohist $=20000$ (Corcuff 2019)

Des modèles inspirés comme celui-ci des comportements d'insectes ou autres animaux dits «sociaux", et plus largement ce que l'on appelle les systèmes d'agents, sont aujourd'hui très présents dans l'architecture et l'urbanisme exploratoires. L'un des défenseurs de ces expérimentations est Neil Leach (Leach 2009), pour ne prendre qu'un exemple.

\section{Conclusion}

Nous n'avons présenté ici que quelques-unes des explorations exposées par Frei Otto dans son ouvrage (Otto 2009). Ces expériences, qu'il s'agisse d' «expériences de pensée » qui s'expriment par des dessins, ou d'expériences impliquant des dispositifs très simples, facilement reproductibles, sont un stimulant pour l'imagination. L'un des objectifs de cet article est d'inciter à lire cet ouvrage, en particulier dans un contexte pédagogique. Même si, rappelons-le, il n'y est aucunement question de numérique, les propositions d'Otto sont autant d'occasions d'imaginer des processus et d'élaborer des algorithmes. L'apprentissage du numérique peut commencer sans ordinateur (Özcar 2005).

D'autre part, ces expériences rencontrent une préoccupation de plus en plus présente dans la recherche en architecture et en urbanisme, que l'on peut résumer sous le terme d'émergence (Hensel et alii 2004). Cette thématique est représentative d'une des évolutions de l'implication du numérique dans la conception architecturale que cette session de SCAN cherche à illustrer.

L'attitude exploratoire que l'exemple de Frei Otto nous invite à adopter instaure une liaison triangulaire entre nature, science et création. La nature, y compris les lois physiques qui induisent la génération de formes, organiques ou non, est la source d'inspiration. L'observation, l'expérimentation, la simulation, y compris numérique, sont les moyens, propres à la science, d'investigation de ces lois de l'espace et des formes. La simulation ouvre sur l'interprétation, l'extrapolation, le détournement, qui mènent à la création.

\section{Bibliographie}

Corcuff, M.-P. (2005). Generative Processes and the Question of Space. In Generative Art 2005 Proceedings, 226-240.

Corcuff, M.-P. (2011). Territories, Boundaries and Connections. In Generative Art 2011

Proceedings, 303-319. 
Corcuff, M.-P., Munch R., Rannou G. (2014). Randomness, (Dis)order and Generativity. In Generative Art 2014 Proceedings, 230-248.

Batty, M., \& Longley, P. (1994). Fractal Cities. London/San Diego : Academic Press Limited

Coates, P. (2010). Programming.Architecture. Abingdon/New York : Routledge.

Hensel, M., Menges, A., Weinstock, M. (2004). Emergence:Morphogenetic Design Strategies. Architectural Design Profile $\mathrm{N}^{\circ} 169$.

Leach, N. (2009). Swarm Urbanism, Architectural Design 79, issue 4, 56-63.

Otto, F. (2009). Occupying and Connecting. Thoughts on Territories and Spheres of Influence with Particular Reference to Human Settlement. Stuttgart/London : Edition Axel Menges.

Özcar, M. (2005). Lesson 1 in Design Computing Does not Have to be with Computers. Basic design exercises, exercises in visual computing. In Digital Design: The Quest for New Paradigms: 23rd eCAADe Conference Proceedings, 311-318.

http://drl.aaschool.ac.uk/portfolio/35-degree/ (consulté le 20 avril 2020)

https://archive.dpa-etsam.com/projects/otto-drops (consulté le 20 avril 2020)

http://www.jbmetais.com/index.php?act=article\&id=22 (consulté le 20 avril 2020) 